\title{
Structure and function of Toll-like receptor 8
}

Umeharu Ohto ${ }^{a}$, Hiromi Tanji ${ }^{a}$, Toshiyuki Shimizu ${ }^{a, b,{ }^{*}}$

${ }^{a}$ Graduate School of Pharmaceutical Sciences, The University of Tokyo, Hongo, Bunkyo-ku,

Tokyo 113-0033, Japan.

${ }^{b}$ CREST, JST.

*Corresponding author. Graduate School of Pharmaceutical Sciences, The University of Tokyo, Hongo, Bunkyo-ku, Tokyo 113-0033, Japan.

Tel.: +81 03-5841-4840; Fax: +81 03-5841-4891; E-mail: shimizu@mol.f.u-tokyo.ac.jp (T. Shimizu). 


\begin{abstract}
Toll-like receptors (TLRs) sense pathogen-associated molecular patterns originating from invading microorganisms and initiate innate immune responses. Recent structural studies of TLR-ligand complexes have revealed the detailed molecular mechanisms by which each TLR specifically recognizes its own ligands. This review focuses on the structure of TLR8 and discusses the similarities and diversities of TLR-ligand interactions and signaling mechanisms.
\end{abstract}

Keywords: Toll like receptor, innate immunity, structural biology. 


\section{Introduction}

Microbial components of invading pathogens, such as lipopeptide, lipopolysaccharide (LPS), and nucleic acids, activate an efficient and immediate innate immune response, followed by acquired immunity [1]. These pathogen-associated molecular patterns (PAMPs) are recognized by a variety of pattern recognition receptors, including Toll-like receptors (TLRs), NOD-like receptors, and RIG-I-like receptors [2]. TLRs, which respond to a wide variety of PAMPs, are type I transmembrane receptors that are characterized by an extracellular domain-containing leucine-rich repeats (LRRs), a transmembrane domain, and a cytoplasmic Toll/Interleukin-1 receptor homology (TIR) domain $[3,4]$. The extracellular LRR domain is responsible for ligand recognition, while the cytoplasmic TIR domain initiates downstream signaling by interacting with other TIR domain-containing adaptor proteins, such as MyD88, MAL, TRIF, and TRAM [5]. The signaling cascades initiated by PAMP-induced stimulation of TLRs lead to a variety of cellular responses (Fig. 1). To date, ten human TLRs have been identified, each of which recognizes a distinct group of PAMPs. Hetero-dimers of TLR2 and TLR1 or TLR6 are activated by lipopeptides [6-9]; TLR3 is activated by viral dsRNA [10]; hetero-dimers of TLR4 and its co-receptor MD-2 are activated by LPS [11-14]; TLR5 is activated by bacterial flagellin [15, 16]; TLR7 and TLR8 are activated by viral or bacterial ssRNA [17-19]; and TLR9 is activated by viral or bacterial CpG DNA [20] (Fig. 1 and Fig. 2). 
Based on their phylogenetic, structural, and functional similarities, TLR7, TLR8 and TLR9 form a subfamily of TLRs. While other human TLRs are expressed on the cell surface, TLR7-9 and TLR3 are localized mainly to endosomal membranes, with the exception of a small population of TLR8 that is also expressed on the cell surface [21]-[22]. Experiments using chimeric receptors comprising the extracellular region of TLR4 and the transmembrane and cytoplasmic regions of other TLRs demonstrated that endosomal membrane targeting is mediated by the transmembrane region and/or intracellular TIR domain [23], [24]. The endoplasmic reticulum membrane protein UNC93B1 is also involved in endosomal targeting of nucleic acid-sensing TLRs [25]. TLR7 and TLR8 share the highest degree of similarity and, since both proteins are receptors for viral ssRNA $[17,19,26]$, their activation is key to the initiation of antiviral responses. Human TLR7 is expressed predominantly in plasmacytoid dendritic cells and B cells, while human TLR8 is expressed in monocytes and myeloid dendritic cells $[27,28]$. The TLR7 and TLR8 signaling pathways are mediated by MyD88; this adaptor protein activates NF-KB, IRF-7, and p38 MAPK, resulting in the induction of proinflammatory cytokines such as tumor necrosis factor $\alpha$, interleukin 6 (IL-6), IL-1 $\beta$, IL-12, and antiviral type I interferons. Murine TLR8 was thought to be non-functional as it was initially observed that TLR7 ${ }^{-/}$mice did not respond to the agonist [29]. However, recent works have demonstrated mouse TLR8 can be activated and play an important role in controlling TLR7 expression [30, 31]. 
Over the last decade, X-ray crystallography studies have made significant contributions to understanding the ligand recognition and signaling mechanisms of TLRs [32-37] (Fig. 3 and Table 1). This review focuses on the function and recently published structure [32] of TLR8, and discusses the similarities and diversities of TLR-ligand interaction and signaling mechanisms.

\section{TLR8 and TLR7 agonists}

ssRNA has been identified as the natural ligand of TLR8 and TLR7 [17, 26]. For example, HIV-1 or influenza virus ssRNAs induce the production of proinflammatory cytokines in plasmacytoid dendritic cells [38]. Although sequence-specific recognition of RNA by TLR7 and TLR8 has not yet been established fully, some sequence preferences have been reported; for example, ssRNAs containing GU-rich or poly-U regions are agonists for both TLR7 and TLR8 [17, 19, 26]. Typical ssRNA agonists are shown in Fig. 4.

TLR7 and TLR8 are also targeted by small chemical compounds with antiviral activity $[29,39]$. The imidazoquinoline derivative imiquimod (R837) is a TLR7-specific agonist, whilst resiquimod (R848) and CL097 are imidazoquinoline-derived TLR8/7 agonists with potent antiviral activity (Fig. 4) [39]. The guanosine analog loxoribine and the pyrimidine analog bropirimine were later found to be TLR7 agonists [40, 41], and CL075 was identified as a thiazoloquinolone derivative that stimulates TLR8 (Fig. 4). Furthermore, many research 
groups have reported the discovery of additional novel TLR8 and TLR7 agonists.

Recent studies have revealed that secreted microRNAs (miRNAs) can be transferred from donor cells to recipient cells, in which they function as ligands of Toll-like receptors [42]. Tumor-secreted miR-21 and miR-29a are recognized by endosomally-expressed human TLR8 or murine TLR7; miRNA-induced activation of these receptors initiates the recruitment of the adaptor molecule MyD88, which drives the production of proinflammatory cytokines such as tumor necrosis factor $\alpha$ and IL-6. These miRNAs harbor a GU-motif that is predominant in ssRNA agonists of TLR8 (or TLR7) (Fig. 4).

\section{Overall structure of the TLR8 ectodomain}

The extracellular domains of TLRs contain 20-26 LRR modules (Fig. 2) and form a horseshoe-shaped right-handed curved solenoid that is characteristic of LRR proteins (Fig. 3 and Fig. 5). Each LRR module comprises 20-30 amino acids; the first ten amino acids form a consensus motif $(x L x x L x L x x N)$ that is highly conserved among LRR proteins, while the remaining portion is variable in sequence and length. Each LRR module represents one turn of the solenoid; the consensus motif forms a $\beta$-strand in the concave face of the horseshoe structure, followed by the variable loop or helical segment forms the ascending lateral, convex, and descending lateral faces (Fig. 5). The $\beta$-strands of flanking LRR modules interact to form a large $\beta$-sheet that spans the whole concave face of the horseshoe structure. While 
typical LRR proteins utilize their concave surface for ligand recognition, such as the binding of ribonuclease A to the concave surface of LRR-containing ribonuclease inhibitor [43], all TLRs whose ligand complexes have been solved utilize their ascending lateral or convex face for ligand recognition (Fig. 3).

The extracellular domains of TLR7, TLR8 and TLR9 comprise approximately 800 amino acids and 26 LRR modules, which is the largest number of LRRs among the human TLRs identified to date (Fig. 2). TLR7-9 contain a characteristic insertion loop (Z-loop) of approximately 30-40 amino acids between LRR14 and LRR15 (Fig. 2). In a recent study, the recombinant full-length ectodomain of human TLR8 expressed in Drosophila melanogaster Schneider 2 cells was cleaved at the Z-loop by an unidentified protease [32]; however, the resulting $\mathrm{N}$-terminal (residues 27-455) and C-terminal (residues 456-827) fragments remained associated during purification, indicating a strong interaction between them. Unlike the horseshoe-shaped structure exhibited by other TLRs, the extracellular domain of human TLR8 forms a ring-shaped structure in which each half of the ring is produced by the $\mathrm{N}$ - and C-terminal fragments (Fig. 3 and Fig. 5). The association between these fragments is mediated by interactions at three distinct regions (Fig. 5A): First, several hydrogen bonds are formed between the N-terminal and C-terminal domains of the protein. Second, the latter half of the Z-loop (residues 458-481) located in the C-terminal fragment forms an ordered structure in the concave surface of the $\mathrm{N}$-terminal fragment by forming extensive hydrophobic 
interactions with LRR3 to LRR18. Note that the first half of the Z-loop (residues 433-457) located in the $\mathrm{N}$-terminal fragment was not present in the electron density map (due to disorder). Third, despite being interrupted by the Z-loop, LRR14 in the N-terminal fragment and LRR15 in the C-terminal fragment form a continuous $\beta$-sheet in the concave face of the ring-like structure.

\section{Proposed mechanism of activation of TLR8}

Based on biochemical and crystallographic studies of TLRs, ligand-induced multimerization of TLR1-6 has been widely accepted (Fig. 3 and Fig. 6). The monomeric extracellular domains of these proteins form homo- or hetero-dimers upon ligand stimulation. TLR2 hetero-dimerizes with TLR1 or TLR6 after binding of triacyl or diacyl lipopeptides, respectively [37], forming a 1:1:1 complex. TLR3 homo-dimerizes after binding of dsRNAs of approximately 50 base pairs, forming a 2:1 TLR3/ligand complex [36]. Additional TLR3 oligomers, such as $3: 1,4: 1$, and 5:1 complexes, can also occur when longer dsRNAs are used as ligands [44]. In collaboration with its co-receptor MD-2, TLR4 homo-dimerizes after binding to LPS, forming a 2:2:2 TLR4/MD-2/LPS complex [34], [45]. TLR5 homo-dimerizes after binding to the bacterial flagellin FliC, forming a 2:2 TLR5/FliC complex [33]. Although the binding interfaces in ligand-induced homo- or hetero-dimers of specific TLRs are considerably different (Fig. 3 and Fig. 7), the overall symmetrical tail-to-tail arrangement of 
two TLRs, in which the C-termini of the TLRs are positioned in the center and the N-termini extend in opposite directions, is very similar. As a result, the C-termini of TLRs within a dimer are in close proximity, which prompts the dimerization of the intracellular TIR domain and subsequent recruitment of the adaptor protein (Fig. 3 and Fig. 6A).

A number of studies have reported that TLR1-6 form dimers in the absence of ligand and that a ligand-induced conformational change in the complex is required for its activation (Fig. 6B). In contrast to the well-established mechanisms involved in ligand-induced multimerization of TLR ectodomains, the events involved in activation of full-length TLRs are less well understood. A recent study using electron microscopy single-particle image reconstruction showed that full-length TLR5 forms an asymmetric homo-dimer in the absence of its ligand flagellin [46], although the interaction between monomers was very weak in solution for the ectodomain constructs used in this study [33]. Using a combination of immunoprecipitation, crosslinking, circular dichroism spectroscopy, and fluorescence resonance energy transfer experiments, Latz et al. showed that TLR9 also forms homo-dimers in the absence of ligand; in this study, the ectodomain of the preformed dimer underwent a conformational change upon ligand binding that resulted in the close apposition of the intracellular TIR domains [47]. Similarly, TLR8 forms a dimer in the absence of ligand and a ligand-induced conformational change is required for its activation [48]. Moreover, several studies have shown that, after their initial ligand-induced homo- or 
hetero-dimerization, TLRs form higher order oligomers in the cell membrane [49-51].

Gel-filtration chromatography and small-angle X-ray scattering analyses of the purified extracellular domain of human TLR8 showed that both the unliganded and liganded forms exist as a dimer [32]. Similar results were also reported for cell-based assays of full-length bovine TLR8 [48] and the dimer formations are supported by the crystal structures of the unliganded and liganded forms of TLR8 (Fig. 8). When viewed from the front, the overall dimer structures of unliganded and liganded TLR8 are similar; both forms exhibit an "m"-shaped tail-to-tail arrangement and both utilize the ascending lateral face as the dimerization interface. However, when viewed from the side, the C-termini of the two protomers are in closer proximity in the liganded form (30 $\AA$ ) than the unliganded form $(50 \AA)$ (Fig. 8). Upon binding of agonistic ligands, the TLR8 dimer undergoes a large structural rearrangement whereby the ligand molecule penetrates the dimerization interface of the unliganded form. Subsequently, the interactions between the two protomers are disrupted and reorganized completely such that the C-termini are brought into close proximity. This structural change enables dimerization of the intracellular TIR domains and subsequent recruitment of the adaptor proteins to initiate downstream signaling cascades (Fig. 6B).

\section{Ligand recognition mechanism of TLR8}

Although their ligand-binding modes display some diversity, all TLRs utilize their 
ascending lateral face for ligand binding (Fig. 3 and Fig. 7). In TLR1, TLR2 and TLR6, the lipopeptide binding site is located in the middle region of the LRR structure [35, 37]. In TLR3, dsRNA binds to two sites located at opposite ends of the LRR structure [36]. The binding sites for LPS-bound MD-2 and FliC in TLR4 and TLR5, respectively, are widely distributed over more than ten LRR units $[33,34]$.

In the TLR8 homo-dimer, the two binding sites for small chemical ligands, such as CL097, CL075 and R848, are located at equivalent positions in the dimerization interface (Fig. 8). Each ligand is sandwiched by the two protomers, TLR8 and TLR8*, and interacts with the N-terminal LRR11-14 of one protomer and the C-terminal LRR16-18 of the other (Fig. 7). Therefore, both the $\mathrm{N}$ - and $\mathrm{C}$-terminal fragments of TLR8 are required for ligand recognition. Specifically, three key interactions occur between TLR8 and its ligands: (i) stacking interactions between the benzene ring of the ligands and Phe405 of TLR8; (ii) hydrogen bonds between the amidine group of the ligands and Asp543 of TLR8, and between the N atoms of the imidazole or thiazole ring of the ligands and Thr574 of TLR8; and (iii) snug fitting of two substituents of the ligand to the small hydrophobic pocket formed between the two protomers (Fig. 9). The importance of the Asp543-mediated interaction to ligand-binding has been demonstrated by a mutagenesis analysis [52]. Interestingly, the residues forming the ligand binding site of TLR8 are well conserved between TLR7 and TLR8 (Fig. 9). Hence, TLR7 would utilize the same ligand binding site as TLR8. The noticeable difference between 
TLR7 and TLR8 is found at the entrance of the ligand binding pocket; TLR8 has an acidic residue Asp545 forming stacking interaction with the aromatic ring of the ligand while TLR7 has a hydrophobic residue Leu557, however how these differences affect ligand specificity is still unknown. Although the binding site in TLR8 for small chemical ligands has been demonstrated by crystallography and subsequently verified by mutagenesis experiments, the binding site for ssRNA remains to be clarified. However, the responsiveness of TLR8 to sSRNA was reduced following mutation of the residues involved in the recognition of small chemical ligands [32], suggesting that this region is also important for the recognition of ssRNA. Because ssRNA and small chemical ligands differ considerably in their sizes and electrostatic properties, further structural analyses are required to confirm this proposal.

\section{Functional implications of processing of the Z-loop}

A number of studies have demonstrated that protease cleavage of the characteristic Z-loop in TLR 7-9 is required for proper functioning of these proteins [53-56]. Ewald et al. were the first to demonstrate processing of the Z-loops in TLR9 and TLR7; this group reported that the ectodomains of these proteins are cleaved in the endolysosome, such that no full-length protein is detectable in the compartment where the ligand is recognized [56]. Several proteases, including asparagine endopeptidase, cathepsin and cysteine protease, are involved in Z-loop cleavage [53-55, 57]. To our knowledge, there are currently no reports 
describing processing of the Z-loop in TLR8, although its functional similarity with TLR7 and TLR9 suggests that similar mechanisms are likely to be involved in the activation of TLR8. While the notion that Z-loop processing is a prerequisite for nucleic acid sensing by TLRs has been widely accepted, the subsequent activation mechanism is a matter of debate, especially with regard to the identity of the functional receptor. For example, Park et al. and Sepulveda et al. reported that, after Z-loop processing, the C-terminal fragment alone is sufficient for ligand binding and the subsequent activation of TLR9 [54, 55]. Conversely, Onji et al. [57] reported that the both the $\mathrm{N}$ - and $\mathrm{C}$-terminal fragments are required for the functional integrity of TLR9 [58]; this latter report is supported by our structural analysis, which revealed that the $\mathrm{N}$ - and C-terminal fragments remain associated after Z-loop cleavage and that both fragments are required for ligand recognition [58].

The end of the $\mathrm{N}$-terminal fragment (Pro432) and the start of the C-terminal fragment (Asp458) of TLR8 are located $44 \AA$ apart and are positioned close to the ascending lateral face involved in dimerization (Fig. 3 and Fig. 8). Given that these two residues are connected by a flexible linker of 26 residues when the Z-loop is unprocessed, the Z-loop likely passes in front of the ascending lateral face of TLR8. An unprocessed Z-loop would interfere with dimerization because there is no space to accommodate the flexible Z-loop between the two protomers in the activated dimer structure of TLR8. The Z-loop sequences in specific TLRs are well conserved across species, while those among the different TLRs within a species are 
not conserved at all; therefore, the features of the Z-loop in TLR8 may not be applicable to TLR7 and TLR9. It is possible that the Z-loop in each TLR plays a distinct role in the regulation of TLR function. Additional crystallographic studies are required to clarify the roles and processing of the Z-loops in individual TLRs.

\section{TLR8 and TLR7 as therapeutic targets}

TLRs are attractive therapeutic targets for the modulation of immune responses and hold promise for the treatment of infection and inflammation. Agonists of TLR7 and TLR8 are currently being considered as promising antiviral and anti-cancer drugs. Imiquimod (marketed as Aldara ${ }^{\circledR}$ by $3 \mathrm{M}$ Pharmaceuticals), one of the first and most successful drugs targeting TLRs, was approved by the US Food and Drug Administration in 1997 for the topical treatment of external genital and perennial warts, actinic keratosis, and non-melanoma skin cancers [59], [60]. Resiquimod, which is structurally related to imiquimod and binds to TLR7 and TLR8, is a considerably more potent analog. This drug is a candidate for treating hepatitis $C$ and other viral infections, and is now being evaluated [61], [62].

Systemic lupus erythematosus is an autoimmune disease that can affect almost any organ system. The disease is characterized by the production of autoantibodies specific for a variety of autoantigens, including nucleic acids, chromatin and ribonucleoproteins. Studies have suggested that the inhibition of immune responses mediated by TLR7, TLR8 and TLR9 
may hold promise for the treatment of systemic lupus erythematosus; therefore, antagonists of TLR7 and TLR8 have been suggested as novel therapeutic targets for the treatment of lupus [63].

\section{Concluding remarks}

Structural studies of the ligand complexes of TLRs have contributed tremendously to current understanding of their diverse ligand recognition and signaling mechanisms. However, the relevance of the activation of distinct signaling pathways by TLRs and the mechanism by which dimerization of the extracellular domains triggers activation of the intracellular TIR domain remain to be addressed. Structural investigation of full-length TLRs, including their transmembrane and intracellular domains, and examination of their interaction with the adaptor proteins will be challenging but will offer a comprehensive understanding of the mechanism of activation TLRs.

\section{Acknowledgments}

This work was supported by a Grant-in-Aid from the Japanese Ministry of Education, Culture, Sports, Science, and Technology (U.O. and T.S.); CREST, JST (T.S.); the Takeda Science Foundation (U.O. and T.S.); and the Mochida Memorial Foundation for Medical and Pharmaceutical Research (U.O.). 


\section{Figure legends}

Fig.1. Schematic illustration of human TLR signaling.

TLR5 and hetero-dimers of TLR2 and TLR1 or TLR6 are expressed at the cell surface, whereas TLR3 and TLR7-9 localize to the endosome where they sense microbial and host-derived nucleic acids. TLR4 localizes to both the plasma membrane and the endosome. TLR signaling is initiated by ligand-induced dimerization of receptors, followed by the engagement of TIR domain-containing adaptor proteins, such as MyD88 and TRIF, which activate downstream signaling cascades. A major consequence of TLR signaling is the induction of proinflammatory cytokines and type I interferons.

Fig. 2. Schematic representation of the extracellular domains of human TLRs. The cognate ligands are shown on the right. The LRRs are numbered and the N-terminal (LRRNT) and C-terminal (LRRCT) domains at the ends of the protein are also indicated. The characteristic Z-loop in TLR7-9 is shown and the LRRs located on either side of the loop are colored differently.

Fig. 3. The activated dimer structures of TLR2/TLR1/Pam3CSK4 (PDB ID: 2Z7X) [37], TLR2/TLR6/Pam2CSK4 (PDB ID: 3A79) [35], TLR4/MD-2/LPS (PDB ID: 3FXI) [34], TLR5/FliC (PDB ID: 3V47) [33], TLR3/dsRNA (PDB ID: 3CIY) [36], and TLR8/CL097 (PDB 
ID: 3W3J) [32]. The color scheme is the same as that used in Figure 2. The ligands are shown in magenta. The gray regions in the TLR2/TLR1/Pam3CSK4, TLR2/TLR6/Pam2CSK4, and TLR5/FliC structures indicate the non-TLR portions. All structural figures were generated using the PyMOL program [64].

Fig. 4. TLR8 and TLR7 agonists.

(A) The sequences of ssRNA agonists and miRNAs that activate human TLR8; ssRNA06 and sSRNA02 contain six repeats of the "UUGU" and "UUAU" motifs, respectively, while ssRNA40 is a phosphorothioate-protected oligonucleotide containing a GU-rich sequence.

(B) The chemical structures of synthetic TLR8 and TLR7 agonists.

Fig. 5. Monomer structures of TLR8 (A) and TLR3 (B) derived from the TLR8/CL097 (PDB ID: 3W3J) [32] and TLR3/dsRNA (PDB ID: 3CIY) [36] complexes, respectively. The LRRs are indicated by underlined numbers, and the $\mathrm{N}$-terminal (LRRNT) and C-terminal (LRRCT) domains at the ends of the protein are shown. The two ligand-binding sites for CL097 are also indicated. The color scheme is the same as that used in Figure 2.

Fig. 6. Mechanisms of TLR activation. 
(A) Ligand-binding induces homo- or hetero-dimerization and activation of TLR1-6 monomers. (B) Ligand-induced conformational changes in preformed dimers are involved in the activation of TLR7-9. The illustration is based on a structural study of TLR8 [32]. Note that structural information about the fate of the intact Z-loop in TLR7-9 is not yet available; therefore, its oligomerization state has not been defined fully. The color scheme is the same as that used in Figure 2.

Fig. 7. Schematic representations of the ligand-binding sites and dimerization interfaces of TLR2/TLR1/Pam3CSK4 (PDB ID: 2Z7X) [37], TLR2/TLR6/Pam2CSK4 (PDB ID: 3A79) [35], TLR4/MD-2/LPS (PDB ID: 3FXI) [34], TLR5/FliC (PDB ID: 3V47) [33], TLR3/dsRNA (PDB ID: 3CIY) [36], and TLR8/CL097 (PDB ID: 3W3J) [32]. The ligands and their binding sites within the TLRs are indicated by red (first ligand) and blue (second ligand) letters and numbers. The red and blue boxes in the TLR4/MD-2/LPS complex represent the binding sites for LPS. Regions missing in the crystal structure are shown in gray. The protein-protein interfaces in the dimer are shown in yellow.

Fig. 8. Dimer structures of the unliganded (PDB ID: 3W3G) and liganded (CL097 complex; PDB ID: 3W3J) forms of TLR8. The color scheme is the same as that used in Figure 2. 
Fig. 9. The ligand-binding site of TLR8 in the TLR8/CL097 complex (PDB ID: 3W3J) [32] (left). Residues interacting with the CL097 molecule are indicated by stick representations. Hydrogen bonds are indicated by with dashed lines. Corresponding residues with the ligand recognition in hTLR7 and hTLR8 are shown (right). Conserved residues are highlighted as red colors. 
Table 1. X-ray crystallographic structures of agonist-induced TLR homo- or hetero-dimers.

\begin{tabular}{|c|c|c|c|c|c|c|}
\hline PDB ID & TLR dimer & Species & Agonist & Resolution & Crystallized regions $^{a}$ & Reference \\
\hline $2 Z 7 X$ & TLR1-TLR2 & Human & $\mathrm{Pam}_{3} \mathrm{CSK}_{4}$ & $2.1 \AA$ & $\begin{array}{l}\text { TLR1 (1-475)_LRR1-18 (18/20), } \\
\text { TLR2 (1-506)_LRR1-19 (19/20) }\end{array}$ & [37] \\
\hline 3A79 & TLR2-TLR6 & Mouse & $\mathrm{Pam}_{2} \mathrm{CSK}_{4}$ & $2.9 \AA$ & $\begin{array}{l}\text { TLR2 (1-506)_LRR1-19 (19/20), } \\
\text { TLR6 (1-482)_LRR1-18 (18/20) }\end{array}$ & [35] \\
\hline $3 \mathrm{ClY}$ & TLR3 & Mouse & $\begin{array}{l}\text { dsRNA } \\
\text { (46mer) }\end{array}$ & $3.4 \AA$ & TLR3 (28-704)_LRR1-23 (23/23) & [36] \\
\hline $3 \mathrm{FXI}$ & TLR4-MD-2 & Human & LPS Ra & $3.1 \AA$ & TLR4 (27-631)_LRR1-22 (22/22) & [34] \\
\hline 3VQ2 & TLR4-MD-2 & Mouse & LPS Re & $2.5 \AA$ & TLR4 (22-627)_LRR1-22 (22/22) & [45] \\
\hline 3VQ1 & TLR4-MD-2 & Mouse & Lipid IVa & $2.7 \AA$ & TLR4 (22-627)_LRR1-22 (22/22) & [45] \\
\hline $4 G 8 A$ & TLR4-MD-2 & Human & LPS $\operatorname{Re}$ & $2.4 \AA$ & $\begin{array}{c}\text { TLR4 (23-629)_LRR1-22 (22/22) } \\
\text { (D299G and T399I) }\end{array}$ & [65] \\
\hline $3 \vee 47$ & TLR5 & Zebrafish & Flagellin & $2.5 \AA$ & TLR5b (22-390)_LRR1-14 (14/22) & [33] \\
\hline $3 W 3 J$ & TLR8 & Human & CL097 & $2.0 \AA$ & TLR8 (27-827)_LRR1-26 (26/26) & [32] \\
\hline $3 W 3 K$ & TLR8 & Human & CL075 & $2.3 \AA$ & TLR8 (27-827)_LRR1-26 (26/26) & [32] \\
\hline $3 W 3 N$ & TLR8 & Human & $\mathrm{R} 848^{\mathrm{b}}$ & $2.1 \AA$ & TLR8 (27-827)_LRR1-26 (26/26) & [32] \\
\hline
\end{tabular}

${ }^{\mathrm{a}}$ The numbers in parentheses after the LRR indicate the number of LRRs in the crystallized construct compared with the number in the entire extracellular region.

${ }^{\mathrm{b}}$ Three structures of the TLR8/R848 complex are deposited in the PDB; however, only one is listed. 


\section{References}

[1] Akira S, Uematsu S, Takeuchi O, Pathogen recognition and innate immunity, Cell 124 (2006) 783-801.

[2] Kawai T, Akira S, The roles of TLRs, RLRs and NLRs in pathogen recognition, Int Immunol 21 (2009) 317-337.

[3] Matsushima N, Tanaka T, Enkhbayar P, Mikami T, Taga M, Yamada K, Kuroki Y, Comparative sequence analysis of leucine-rich repeats (LRRs) within vertebrate toll-like receptors, BMC genomics 8 (2007) 124.

[4] Bell JK, Mullen GE, Leifer CA, Mazzoni A, Davies DR, Segal DM, Leucine-rich repeats and pathogen recognition in Toll-like receptors, Trends Immunol 24 (2003) 528-533.

[5] Kawai T, Akira S, The role of pattern-recognition receptors in innate immunity: update on Toll-like receptors, Nat Immunol 11 (2010) 373-384.

[6] Takeuchi O, Kawai T, Muhlradt PF, Morr M, Radolf JD, Zychlinsky A, Takeda K, Akira S, Discrimination of bacterial lipoproteins by Toll-like receptor 6, Int Immunol 13 (2001) 933-940.

[7] Takeuchi O, Sato S, Horiuchi T, Hoshino K, Takeda K, Dong ZY, Modlin RL, Akira S, Cutting edge: Role of Toll-like receptor 1 in mediating immune response to microbial lipoproteins, J Immunol 169 (2002) 10-14.

[8] Alexopoulou L, Thomas V, Schnare M, Lobet Y, Anguita J, Schoen RT, Medzhitov R, Fikrig E, Flavell RA, Hyporesponsiveness to vaccination with Borrelia burgdorferi OspA in humans and in TLR1- and TLR2-deficient mice, Nat Med 8 (2002) 878-884.

[9] Ozinsky A, Underhill DM, Fontenot JD, Hajjar AM, Smith KD, Wilson CB, Schroeder L, Aderem A, The repertoire for pattern recognition of pathogens by the innate immune system is defined by cooperation between Toll-like receptors, Proc Natl Acad Sci USA 97 (2000) 13766-13771.

[10] Alexopoulou L, Holt AC, Medzhitov R, Flavell RA, Recognition of double-stranded RNA and activation of NF-kappa B by Toll-like receptor 3, Nature 413 (2001) 732-738.

[11] Poltorak A, He XL, Smirnova I, Liu MY, Van Huffel C, Du X, Birdwell D, Alejos E, Silva M, Galanos C, Freudenberg M, Ricciardi-Castagnoli P, Layton B, Beutler B, Defective LPS signaling in C3H/HeJ and C57BL/10ScCr mice: Mutations in Tlr4 gene, Science 282 (1998) 2085-2088.

[12] Qureshi ST, Lariviere L, Leveque G, Clermont S, Moore KJ, Gros P, Malo D, Endotoxin-tolerant mice have mutations in toll-like receptor 4 (Tlr4), J Exp Med 189 (1999) 615-625.

[13] Hoshino K, Takeuchi O, Kawai T, Sanjo H, Ogawa T, Takeda Y, Takeda K, Akira S, Cutting edge: Toll-like receptor 4 (TLR4)-deficient mice are hyporesponsive to lipopolysaccharide: Evidence for TLR4 as the Lps gene product, J Immunol 162 (1999) 3749-3752.

[14] Shimazu R, Akashi S, Ogata H, Nagai Y, Fukudome K, Miyake K, Kimoto M, MD-2, a molecule that confers lipopolysaccharide responsiveness on Toll-like receptor 4, J Exp Med 189 (1999) 1777-1782.

[15] Hayashi F, Smith KD, Ozinsky A, Hawn TR, Yi EC, Goodlett DR, Eng JK, Akira S, Underhill DM, Aderem A, The innate immune response to bacterial flagellin is mediated by Toll-like receptor 5, Nature 410 
(2001) 1099-1103.

[16] Uematsu S, Jang MH, Chevrier N, Guo ZJ, Kumagai Y, Yamamoto M, Kato H, Sougawa N, Matsui H, Kuwata H, Hemmi H, Coban C, Kawai T, Ishii KJ, Takeuchi O, Miyasaka M, Takeda K, Akira S, Detection of pathogenic intestinal bacteria by Toll-like receptor 5 on intestinal CD11c(+) lamina propria cells, Nat Immunol 7 (2006) 868-874.

[17] Heil F, Hemmi H, Hochrein H, Ampenberger F, Kirschning C, Akira S, Lipford G, Wagner H, Bauer S, Species-specific recognition of single-stranded RNA via toll-like receptor 7 and 8, Science 303 (2004) 1526-1529.

[18] Diebold SS, Kaisho T, Hemmi H, Akira S, Sousa CRE, Innate antiviral responses by means of TLR7-mediated recognition of single-stranded RNA, Science 303 (2004) 1529-1531.

[19] Lund JM, Alexopoulou L, Sato A, Karow M, Adams NC, Gale NW, Iwasaki A, Flavell RA, Recognition of single-stranded RNA viruses by Toll-like receptor 7, Proc Natl Acad Sci USA 101 (2004) 5598-5603.

[20] Hemmi H, Takeuchi O, Kawai T, Kaisho T, Sato S, Sanjo H, Matsumoto M, Hoshino K, Wagner H, Takeda K, Akira S, A Toll-like receptor recognizes bacterial DNA, Nature 408 (2000) 740-745.

[21] Lee J, Chuang TH, Redecke V, She LP, Pitha PM, Carson DA, Raz E, Cottam HB, Molecular basis for the immunostimulatory activity of guanine nucleoside analogs: Activation of Toll-like receptor 7, Proc Natl Acad Sci USA 100 (2003) 6646-6651.

[22] Miggin SM, O'Neill LAJ, New insights into the regulation of TLR signaling, J Leukocyte Biol 80 (2006) 220-226.

[23] Nishiya T, DeFranco AL, Ligand-regulated chimeric receptor approach reveals distinctive subcellular localization and signaling properties of the toll-like receptors, J Biol Chem 279 (2004) 19008-19017.

[24] Nishiya T, Kajita E, Miwa S, DeFranco AL, TLR3 and TLR7 are targeted to the same intracellular compartments by distinct regulatory elements, J Biol Chem 280 (2005) 37107-37117.

[25] Kim YM, Brinkmann MM, Paquet ME, Ploegh HL, UNC93B1 delivers nucleotide-sensing toll-like receptors to endolysosomes, Nature 452 (2008) 234-U280.

[26] Diebold SS, Kaisho T, Hemmi H, Akira S, Reis e Sousa C, Innate antiviral responses by means of TLR7-mediated recognition of single-stranded RNA, Science 303 (2004) 1529-1531.

[27] Hornung V, Rothenfusser S, Britsch S, Krug A, Jahrsdorfer B, Giese T, Endres S, Hartmann G, Quantitative expression of toll-like receptor 1-10 mRNA in cellular subsets of human peripheral blood mononuclear cells and sensitivity to CpG oligodeoxynucleotides, J Immunol 168 (2002) 4531-4537.

[28] Marques JT, Williams BRG, Activation of the mammalian immune system by siRNAs, Nat Biotechnol 23 (2005) 1399-1405.

[29] Jurk M, Heil F, Vollmer J, Schetter C, Krieg AM, Wagner H, Lipford G, Bauer S, HumanTLR7 orTLR8 independently confer responsiveness to the antiviral compound R-848, Nat Immunol 3 (2002) 499-499.

[30] Cervantes JL, Weinerman B, Basole C, Salazar JC, TLR8: the forgotten relative revindicated, Cell Mol Immunol 9 (2012) 434-438.

[31] Demaria O, Pagni PP, Traub S, de Gassart A, Branzk N, Murphy AJ, Valenzuela DM, Yancopoulos 
GD, Flavell RA, Alexopoulou L, TLR8 deficiency leads to autoimmunity in mice, J Clin Invest 120 (2010) 3651-3662.

[32] Tanji H, Ohto U, Shibata T, Miyake K, Shimizu T, Structural reorganization of the Toll-like receptor 8 dimer induced by agonistic ligands, Science 339 (2013) 1426-1429.

[33] Yoon SI, Kurnasov O, Natarajan V, Hong M, Gudkov AV, Osterman AL, Wilson IA, Structural basis of TLR5-flagellin recognition and signaling, Science 335 (2012) 859-864.

[34] Park BS, Song DH, Kim HM, Choi BS, Lee H, Lee JO, The structural basis of lipopolysaccharide recognition by the TLR4-MD-2 complex, Nature 458 (2009) 1191-1195.

[35] Kang JY, Nan X, Jin MS, Youn SJ, Ryu YH, Mah S, Han SH, Lee H, Paik SG, Lee JO, Recognition of lipopeptide patterns by Toll-like receptor 2-Toll-like receptor 6 heterodimer, Immunity 31 (2009) 873-884.

[36] Liu L, Botos I, Wang Y, Leonard JN, Shiloach J, Segal DM, Davies DR, Structural basis of toll-like receptor 3 signaling with double-stranded RNA, Science 320 (2008) 379-381.

[37] Jin MS, Kim SE, Heo JY, Lee ME, Kim HM, Paik SG, Lee H, Lee JO, Crystal structure of the TLR1-TLR2 heterodimer induced by binding of a tri-acylated lipopeptide, Cell 130 (2007) 1071-1082.

[38] Alter G, Suscovich TJ, Teigen N, Meier A, Streeck H, Brander C, Altfeld M, Single-stranded RNA derived from HIV-1 serves as a potent activator of NK cells, J Immunol 178 (2007) 7658-7666.

[39] Hemmi H, Kaisho T, Takeuchi O, Sato S, Sanjo H, Hoshino K, Horiuchi T, Tomizawa H, Takeda K, Akira S, Small anti-viral compounds activate immune cells via the TLR7 MyD88-dependent signaling pathway, Nat Immunol 3 (2002) 196-200.

[40] Lee J, Chuang TH, Redecke V, She L, Pitha PM, Carson DA, Raz E, Cottam HB, Molecular basis for the immunostimulatory activity of guanine nucleoside analogs: activation of Toll-like receptor 7, Proc Natl Acad Sci USA 100 (2003) 6646-6651.

[41] Heil F, Ahmad-Nejad P, Hemmi H, Hochrein H, Ampenberger F, Gellert T, Dietrich H, Lipford G, Takeda K, Akira S, Wagner H, Bauer S, The Toll-like receptor 7 (TLR7)-specific stimulus loxoribine uncovers a strong relationship within the TLR7, 8 and 9 subfamily, Eur J Immunol 33 (2003) 2987-2997.

[42] Fabbri M, Paone A, Calore F, Galli R, Gaudio E, Santhanam R, Lovat F, Fadda P, Mao C, Nuovo GJ, Zanesi N, Crawford M, Ozer GH, Wernicke D, Alder H, Caligiuri MA, Nana-Sinkam P, Perrotti D, Croce CM, MicroRNAs bind to Toll-like receptors to induce prometastatic inflammatory response, Proc Natl Acad Sci USA 109 (2012) E2110-2116.

[43] Kobe B, Deisenhofer J, Mechanism of ribonuclease inhibition by ribonuclease inhibitor protein based on the crystal structure of its complex with ribonuclease A, J Mol Biol 264 (1996) 1028-1043.

[44] Leonard JN, Ghirlando R, Askins J, Bell JK, Margulies DH, Davies DR, Segal DM, The TLR3 signaling complex forms by cooperative receptor dimerization, Proc Natl Acad Sci USA 105 (2008) 258-263.

[45] Ohto U, Fukase K, Miyake K, Shimizu T, Structural basis of species-specific endotoxin sensing by innate immune receptor TLR4/MD-2, Proc Natl Acad Sci USA 109 (2012) 7421-7426.

[46] Zhou KF, Kanai R, Lee P, Wang HW, Modis Y, Toll-like receptor 5 forms asymmetric dimers in the absence of flagellin, J Struct Biol 177 (2012) 402-409.

[47] Latz E, Verma A, Visintin A, Gong M, Sirois CM, Klein DC, Monks BG, McKnight CJ, Lamphier 
MS, Duprex WP, Espevik T, Golenbock DT, Ligand-induced conformational changes allosterically activate Toll-like receptor 9, Nat Immunol 8 (2007) 772-779.

[48] Zhu J, Brownlie R, Liu Q, Babiuk LA, Potter A, Mutwiri GK, Characterization of bovine Toll-like receptor 8: ligand specificity, signaling essential sites and dimerization, Mol Immunol 46 (2009) 978-990.

[49] Luo JQ, Obmolova G, Malia TJ, Wu SJ, Duffy KE, Marion JD, Bell JK, Ge P, Zhou ZH, Teplyakov A, Zhao YH, Lamb RJ, Jordan JL, San Mateo LR, Sweet RW, Gilliland GL, Lateral clustering of TLR3:dsRNA signaling units revealed by TLR3ecd:3Fabs quaternary structure, J Mol Biol 421 (2012) 112-124.

[50] Lin SC, Lo YC, Wu H, Helical assembly in the MyD88-IRAK4-IRAK2 complex in TLR/IL-1R signalling, Nature 465 (2010) 885-U882.

[51] Visintin A, Latz E, Monks BG, Espevik T, Golenbock DT, Lysines 128 and 132 enable lipopolysaccharide binding to MD-2, leading to Toll-like receptor-4 aggregation and signal transduction, J Biol Chem 278 (2003) 48313-48320.

[52] Gibbard RJ, Morley PJ, Gay NJ, Conserved features in the extracellular domain of human toll-like receptor 8 are essential for pH-dependent signaling, J Biol Chem 281 (2006) 27503-27511.

[53] Ewald SE, Engel A, Lee J, Wang MQ, Bogyo M, Barton GM, Nucleic acid recognition by Toll-like receptors is coupled to stepwise processing by cathepsins and asparagine endopeptidase, J Exp Med 208 (2011) 643-651

[54] Sepulveda FE, Maschalidi S, Colisson R, Heslop L, Ghirelli C, Sakka E, Lennon-Dumenil AM, Amigorena S, Cabanie L, Manoury B, Critical role for asparagine endopeptidase in endocytic Toll-like receptor signaling in dendritic cells, Immunity 31 (2009) 737-748.

[55] Park B, Brinkmann MM, Spooner E, Lee CC, Kim YM, Ploegh HL, Proteolytic cleavage in an endolysosomal compartment is required for activation of Toll-like receptor 9, Nat Immunol 9 (2008) 1407-1414.

[56] Ewald SE, Lee BL, Lau L, Wickliffe KE, Shi GP, Chapman HA, Barton GM, The ectodomain of Toll-like receptor 9 is cleaved to generate a functional receptor, Nature 456 (2008) 658-U688.

[57] Asagiri M, Hirai T, Kunigami T, Kamano S, Gober HJ, Okamoto K, Nishikawa K, Latz E, Golenbock DT, Aoki K, Ohya K, Imai Y, Morishita Y, Miyazono K, Kato S, Saftig P, Takayanagi H, Cathepsin K-dependent toll-like receptor 9 signaling revealed in experimental arthritis, Science 319 (2008) 624-627.

[58] Onji M, Kanno A, Saitoh SI, Fukui R, Motoi Y, Shibata T, Matsumoto F, Lamichhane A, Sato S, Kiyono H, Yamamoto K, Miyake K, An essential role for the N-terminal fragment of Toll-like receptor 9 in DNA sensing, Nat Commun 4 (2013) 1949-1958.

[59] Stockfleth E, Christophers E, Benninghoff B, Sterry W, Low incidence of new actinic keratoses after topical 5\% imiquimod cream treatment: a long-term follow-up study, Arch Dermatol 140 (2004) 1542.

[60] Somani N, Rivers JK, Imiquimod 5\% cream for the treatment of actinic keratoses, Skin Therapy Lett 10 (2005) 1-6.

[61] Mark KE, Corey L, Meng TC, Magaret AS, Huang ML, Selke S, Slade HB, Tyring SK, Warren T, Sacks SL, Leone P, Bergland VA, Wald A, Topical resiquimod 0.01\% gel decreases herpes simplex virus type 2 genital shedding: A randomized, controlled trial, J Infect Dis 195 (2007) 1324-1331.

[62] Pockros PJ, Guyader D, Patton H, Tong MJ, Wright T, McHutchison JG, Meng TC, Oral resiquimod 
in chronic HCV infection: Safety and efficacy in 2 placebo-controlled, double-blind phase IIa studies, J Hepatol 47 (2007) 174-182.

[63] Barrat FJ, Meeker T, Gregorio J, Chan JH, Uematsu S, Akira S, Chang B, Duramad O, Coffman RL, Nucleic acids of mammalian origin can act as endogenous ligands for Toll-like receptors and may promote systemic lupus erythematosus, J Exp Med 202 (2005) 1131-1139.

[64] DeLano WL, The PyMOL Molecular Graphics System. DeLano Scientific LLC, Palo Alto, CA, USA. http://www.pymol.org., (2008).

[65] Ohto U, Yamakawa N, Akashi-Takamura S, Miyake K, Shimizu T, Structural analyses of human Toll-like receptor 4 polymorphisms D299G and T399I, J Biol Chem 287 (2012) 40611-40617. 